\title{
LSH Based Outlier Detection and Its Application in Distributed Setting
}

\author{
Madhuchand Rushi Pillutla, Nisarg Raval, Piyush Bansal, \\ Kannan Srinathan and C. V. Jawahar \\ International Institute of Information Technology Hyderabad, India \\ \{rushi.pillutla, nisarg.raval, piyush_bansal\}@ research.iiit.ac.in \\ \{srinathan, jawahar\}@iiit.ac.in
}

\begin{abstract}
In this paper, we give an approximate algorithm for distance based outlier detection using Locality Sensitive Hashing (LSH) technique. We propose an algorithm for the centralized case wherein the entire dataset is locally available for processing. However, in case of very large datasets collected from various input sources, often the data is distributed across the network. Accordingly, we show that our algorithm can be effectively extended to a constant round protocol with low communication costs, in a distributed setting with horizontal partitioning.
\end{abstract}

\section{Categories and Subject Descriptors}

H.2.8 [Database Management]: Database Applications

\section{General Terms}

Algorithms, Performance

\section{INTRODUCTION}

Our outlier detection scheme is based on the definition introduced by Knorr et al. [2], which says that an object $o$ is an outlier if a very large fraction $p_{t}$ of the total objects in the dataset $D$ lie outside the radius $d_{t}$ from $o$. In our approach, we use the converse of this idea and say an object to be a non-outlier if it has enough neighbors within the radius $d_{t}$. This way, all those non-outliers in the data which have many near neighbors can be identified easily, without calculating the distances to every other object in the dataset. Moreover, in our approach whenever we identify a non-outlier we will be able to say most of its neighbors as non-outliers without even considering those objects separately. Thus, we obtain a very efficient pruning technique where, most of the nonoutliers in the dataset can be easily pruned and a very small percent of the objects in the dataset need to be processed

*This work was partly supported by Naval Research Board, India.

Permission to make digital or hard copies of all or part of this work for personal or classroom use is granted without fee provided that copies are not made or distributed for profit or commercial advantage and that copies bear this notice and the full citation on the first page. To copy otherwise, to republish, to post on servers or to redistribute to lists, requires prior specific permission and/or a fee.

CIKM'11, October 24-28, 2011, Glasgow, Scotland, UK.

Copyright 2011 ACM 978-1-4503-0717-8/11/10 ...\$10.00. after pruning. In order to efficiently find the near neighbors in a large dimensional data, we use the Locality Sensitive Hashing(LSH) scheme. The idea of Locality Sensitive Hashing was first introduced in [1]. The basic concept of LSH is to hash all the objects such that similar objects are hashed to the same bin with high probability. Mathematically, this idea is formalized as follows [1]:

Definition 1. A family $H=h: S \rightarrow U$ is called $\left(r_{1}, r_{2}\right.$, $\left.p_{1}, p_{2}\right)$ - sensitive if for any two objects $\mathrm{p}, \mathrm{q}$ in $\mathrm{S}$ :

$$
\begin{aligned}
& \text { if } d(p, q) \leq r_{1}: \operatorname{Pr}[h(p)=h(q)] \geq p_{1} \\
& \text { if } d(p, q) \geq r_{2}: \operatorname{Pr}[h(p)=h(q)] \leq p_{2}
\end{aligned}
$$

where $d(p, q)$ is the distance between objects $p$ and $q$. For the hashing scheme to be locality sensitive, two conditions to be satisfied are $r_{2}>r_{1}$ and $p_{2}<p_{1}$. In order to amplify the gap between the probabilities $p_{1}$ and $p_{2}$, standard practice is to concatenate several hash functions to obtain a function family $G=\left\{g: S \rightarrow U^{k}\right\}$ such that $g(p)=\left(h_{1}(p), h_{2}(p), \ldots, h_{k}(p)\right)$; where $k$ is the width of each hash function and $h_{i} \in H$. For a hash function family $G$, the probabilities in Equation 1 and 2 are modified as:

$$
\begin{aligned}
& \text { if } d(p, q) \leq r_{1}: \operatorname{Pr}[g(p)=g(q)] \geq p_{1}^{k} \\
& \text { if } d(p, q) \geq r_{2}: \operatorname{Pr}[g(p)=g(q)] \leq p_{2}^{k}
\end{aligned}
$$

During LSH, each object $o \in D$ is stored in the bins $g_{j}(o)$ for $j=1,2 \ldots L$; where each $g$ is drawn independently and uniformly at random from $G$ i.e., each object is hashed using $L$ hash functions drawn from $G$ and stored in the corresponding bins. Recently Wang et al. [4] proposed an outlier detection method using LSH, but unlike our approach their scheme uses the definition of an outlier given by [3].

\section{ALGORITHMS}

In this section we describe our algorithms for both centralized as well as distributed scheme in detail.

\subsection{Centralized Setting}

The algorithm for outlier detection in the centralized setting takes as input the dataset $D$, distance threshold $d_{t}$, point threshold $p_{t}$ and outputs the outlier set $M$. The algorithm for centralized setting is executed in three phases.

In the First phase, initially the parameters $R=r_{1}=$ $d_{t} /(1+\epsilon)$ and $p_{t}^{\prime}=\left(1-p_{t}\right) \times|D|$ are computed. Here $\epsilon>0$ is an approximation factor. The LSH scheme is applied on the dataset with the parameter $\mathrm{R}$, the output of which is an $L \times H$ array of bins with each bin containing the set of objects hashed to that bin. In the Second phase ApproximateOD 

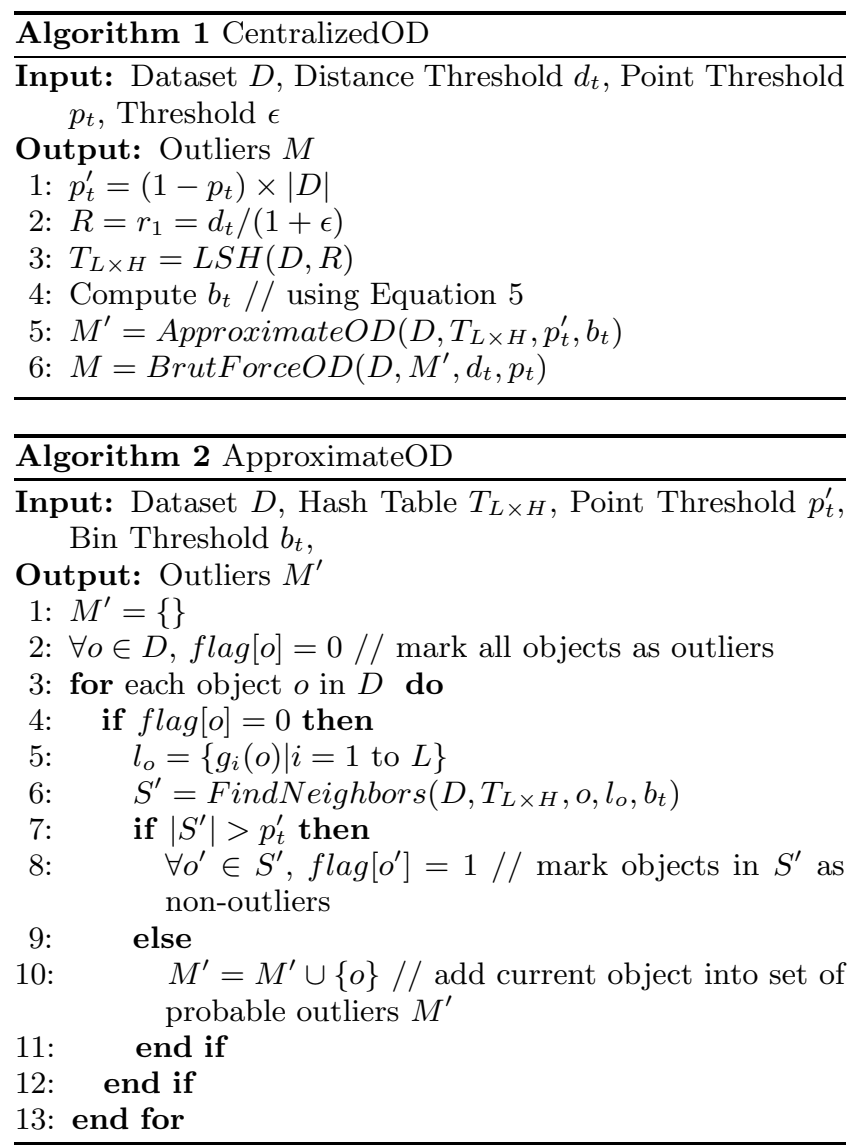

(Algorithm 2), using the LSH binning structure, most of the non-outliers in the dataset are pruned. We consider an object $o$ and find all $L$ bins to which it is hashed by LSH. We then construct the set $S$ of all the objects stored in those $L$ bins (without removing duplicates). The objects in this set are the probable neighbors of $o$. More precisely, from Equation 4, we know that each object in $S$ is within the distance $r_{2}=(1+\epsilon) \times r_{1}=d_{t}$ from $o$, with a probability at least $\left(1-p_{2}^{k}\right)$. To boost this probability, we consider only those objects which are repeated at least $b_{t}\left(b_{t} \leq L\right)$ times in the $L$ bins and store only those objects in a new set $S^{\prime}$. In other words, we are reducing the error probability

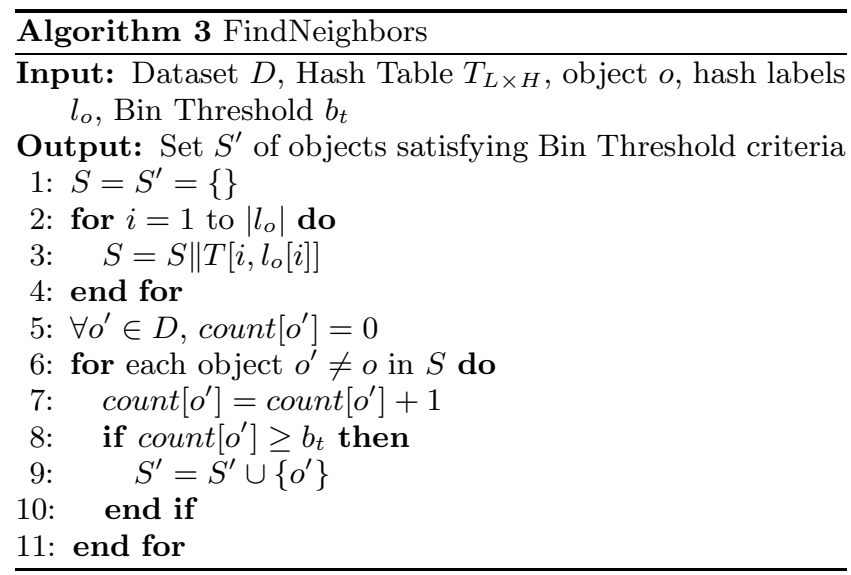

of considering an actual non-neighbor as a neighbor of the object $o$. Here, $b_{t}$ is a bin threshold which can be computed based on the desired false negative probability as explained latter in this section.

If the cardinality of $S^{\prime}$, returned by the FindNeighbors (Algorithm 3) protocol is greater than the modified point threshold $p_{t}^{\prime}=\left(1-p_{t}\right) \times|D|$, with a very high probability $o$ cannot be an outlier since it has sufficient neighbors within distance $d_{t}$. Moreover, this holds true for all the objects in $S^{\prime}$ i.e., every object other than $o$ also has more than $p_{t}^{\prime}$ neighbors with in the distance $d_{t}$, so it can not be an outlier (with a very high probability). Hence, all the objects in $S^{\prime}$ are marked as non outliers. If, on the other hand the cardinality of $S^{\prime}$ is less than or equal to the point threshold $p_{t}^{\prime}$, we consider the object $o$ as a probable outlier. This procedure is repeated till all the objects are either marked as non-outliers or probable outliers. We denote the number of objects for which the FindNeighbors protocol is queried as $N_{q}$ and in the Section 3, we show that this value is very less (less than 1\%) compared to the total dataset size. The resulting set of probable outliers $M^{\prime}$ can have a few false positives and negligible amount of false negatives. In the final phase BruteForceOD (Algorithm 4), the false positives are removed and the final set of approximate outliers $M$ is returned.

The overall computational complexity of Algorithm 1 is $O(n d L)$; where $n=|D|, d$ is the dimensionality of the dataset and $L=n^{1 /(1+\epsilon)}$.

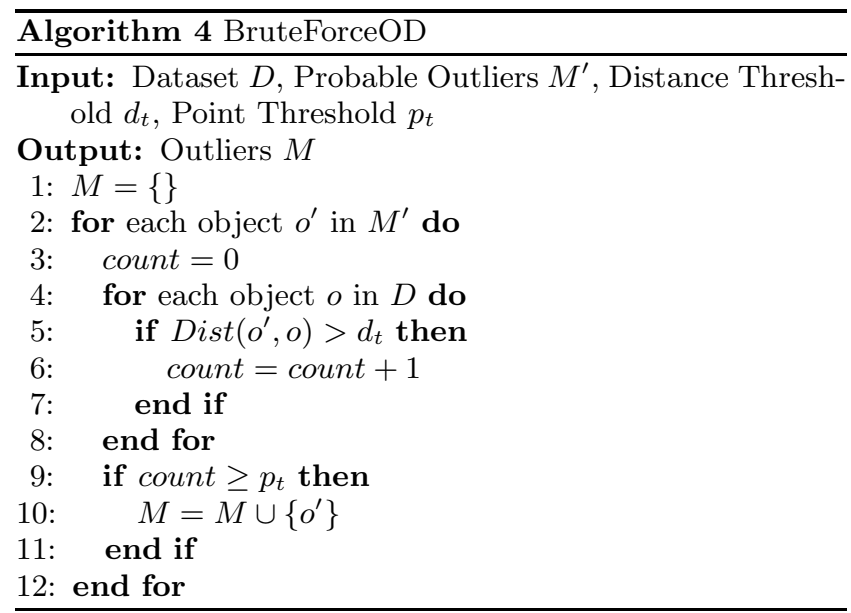

False Positives and False Negatives: In the context of outlier detection, a false positive $(f p)$ is to label a nonoutlier as an outlier and false negative $(f n)$ is to label an outlier to be a non-outlier. In this section we give a bound on the probabilities of both based on the value of the bin threshold parameter $b_{t}$. Consider a LSH scheme where each object is hashed using $\mathrm{L}$ hash functions each of width $\mathrm{k}$. The probability of two objects at a distance greater than $r_{2}=r_{1} \times(1+\epsilon)=d_{t}$, to be hashed to the same bin is at most $p_{2}^{k}$. Consider the worst case scenario where an actual outlier has exactly $p_{t}^{\prime}$ neighbors. Hence, counting one nonneighbor as a neighbor will lead to a false negative. In our scheme, for a non-neighbor to be counted as a neighbor of an object $o$, it should hash to the same bin as $o$ for at least $b_{t}$ times out of $L$ times. The probability that this happens for exactly $b_{t}$ times out of $L$ is given by the binomial probability:

$$
\operatorname{Pr}^{\prime}[f n] \leq\left(\begin{array}{c}
L \\
b_{t}
\end{array}\right) P_{2}^{k b_{t}}\left(1-P_{2}^{k b_{t}}\right)^{L-b_{t}}
$$


Hence, probability for a false negative is upper bounded by:

$$
\operatorname{Pr}[f n]<\left(L-b_{t}\right) \times\left(\begin{array}{c}
L \\
b_{t}
\end{array}\right) P_{2}^{k b_{t}}\left(1-P_{2}^{k b_{t}}\right)^{L-b_{t}}
$$

Similarly, the probability that two objects within a distance $\mathrm{R}$ being hashed to two different bins is at most $\left(1-p_{1}^{k}\right)$. The probability that this will happen at least $\left(L-b_{t}\right)$ times out of $L$ times is given as:

$$
\operatorname{Pr}[f p]<b_{t} \times\left(\begin{array}{c}
L \\
L-b_{t}
\end{array}\right)\left(1-p_{1}^{k\left(L-b_{t}\right)}\right) p_{1}^{k b_{t}}
$$

This gives an upper bound for the probability of a false positive.

Bin Threshold: As seen from the Equations 5 and 6, both the false negative and false positive probabilities depend on the bin threshold. Increasing the bin threshold has an effect of decreasing the false negatives at the cost of an increase in the false positives and vise versa. An optimal value for $b_{t}$ would be one which would remove the false negatives at the cost of introducing minimal false positives. In our scheme, the user has the flexibility to fix $b_{t}$, based on the false negative probability desired, using Equations 5 .

\subsection{Distributed Setting}

We consider the horizontal distribution where each player has a subset of the total number of objects. The distributed algorithm is given in two player setting, which can be easily extended to a $p$ player setting. Consider two players denoted by $P^{A}$ and $P^{B}$ with local datasets $D^{A}$ and $D^{B}$ respectively.

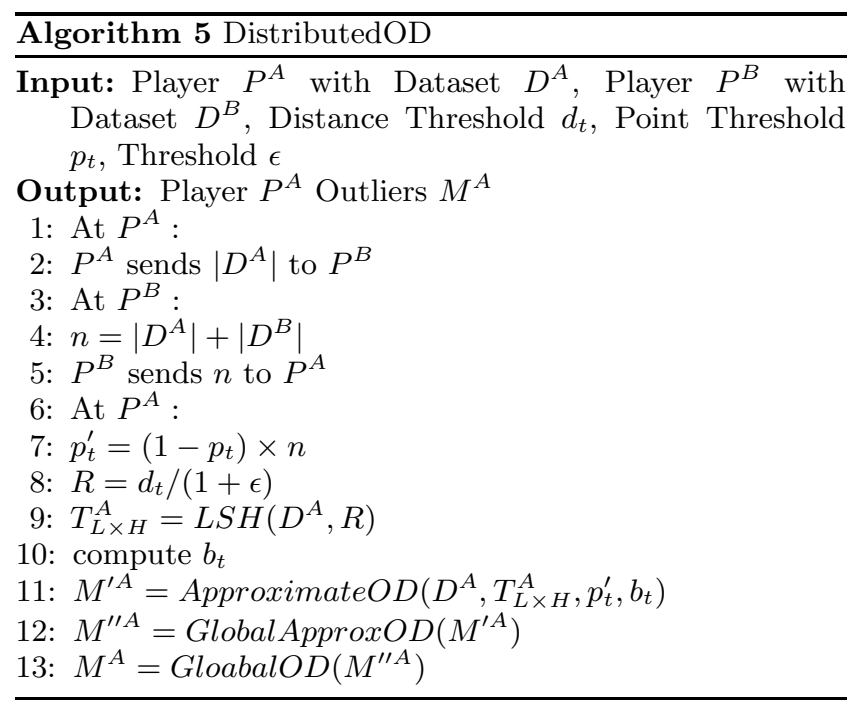

We present the algorithm such that one player, say $P^{A}$ will be able to compute its subset of the global outliers at the end of the algorithm. Similarly the algorithm can be used to enable $P^{B}$ to compute its subset of the global outliers by simply interchanging the roles of $P^{A}$ and $P^{B}$ in the algorithm. Before giving a detailed description of the algorithm, we give the following definitions.

Definition 2. local outlier: Given a distance threshold $d_{t}$ and a point threshold $p_{t}$, an object $o$ with player $P^{i}$ is a local outlier if the number of objects in the local dataset $D^{i}$ lying at a distance greater than $d_{t}$ is at least a fraction $p_{t}$ of the total dataset $D$.
Definition 3. global outlier: Given a distance threshold $d_{t}$ and a point threshold $p_{t}$, an object $o$ in a dataset $D$ is a global outlier if at least fraction $p_{t}$ of the objects in $D$ lie at a distance greater than $d_{t}$ from $o$.

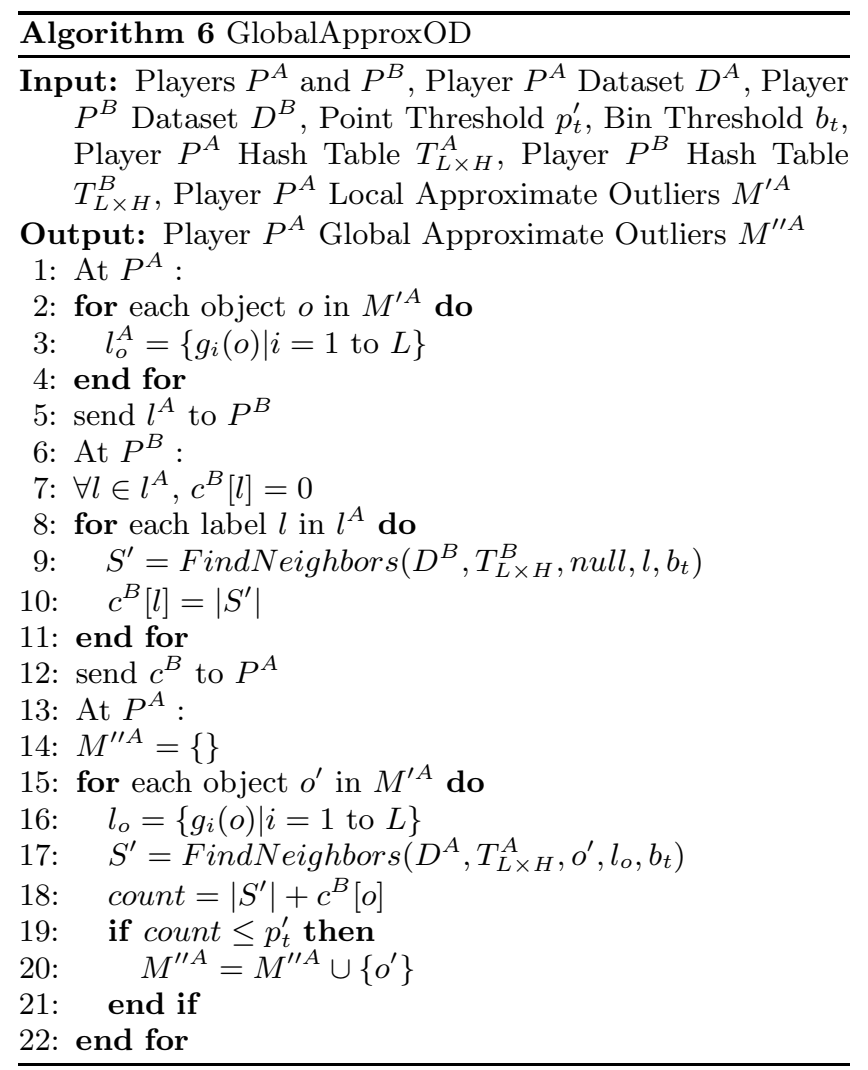

In the first phase, player $P^{A}$ sends the size of his dataset to $P^{B}$ and gets the size of the entire dataset (i.e. $\left|D^{A}\right|+\left|D^{B}\right|$ ). Player $P^{A}$ locally computes its local probable outliers $M^{\prime A}$ by running the centralized algorithm on its dataset $D^{A}$. In the second phase, for each object $o$ in the set $M^{\prime A}, P^{A}$ forms the set $l_{o}^{A}$ which is the set of $\mathrm{L}$ labels of the bins to which $o$ is hashed to (these labels are stored while performing LSH). $P^{A}$ sends the set $l^{A}$ to $P^{B}$. Player $P^{B}$ considers each element $l$ in $l^{A}$ and runs the FindNeighbors algorithm on its local dataset. $P^{B}$ then counts the cardinality of the sets returned by FindNeighbors and sends the set of counts to $P^{A}$. Player $P^{A}$ considers each object $o$ in $M^{\prime A}$ and runs the FindNeighbors algorithm to obtain its count of the number of objects repeating more than $b_{t}$ times (this step is actually redundant if $P^{A}$ stores this count in first phase). $P^{A}$ then computes the sum of this count and the corresponding count in $c^{B}$, and if this sum is less than or equal to point threshold $P_{t}^{\prime}, P^{A}$ stores $O$ in the set $M^{\prime \prime A}$ of global probable outliers.

The set $M^{\prime \prime A}$ contains some false positives which can be removed in third phase with another round of communication as follows: $P^{A}$ sends the set $M^{\prime \prime A}$ to $P^{B}$. Player $P^{B}$ considers each object $o$ in $M^{\prime \prime A}$ and computes the distance to each object in its dataset $D^{B}$ and counts the number of objects which lie at distance greater than the distance threshold $d_{t}$ from $o . P^{B}$ sends all the counts back to $P^{A}$. $P^{A}$ then considers each object $o$ in $M^{\prime \prime A}$ and computes the distance to each object in its dataset $D^{A}$ and counts the number of objects lying at distance greater than $d_{t} . P^{A}$ 
then computes the sum of this count and the corresponding count received from $P^{B}$ and if this count is greater than or equal to fraction $p_{t}$ of $n$, marks the object $o$ as an outlier.

The computational complexity of player $P^{A}$ is $O\left(n^{A} d L\right)$. The overall communication complexity of player $P^{A}$ would be $O\left(m^{\prime A} L+m^{\prime \prime A} d\right)$, where $m^{\prime A}=\left|M^{\prime A}\right|$ and $m^{\prime \prime A}=\left|M^{\prime \prime A}\right|$.

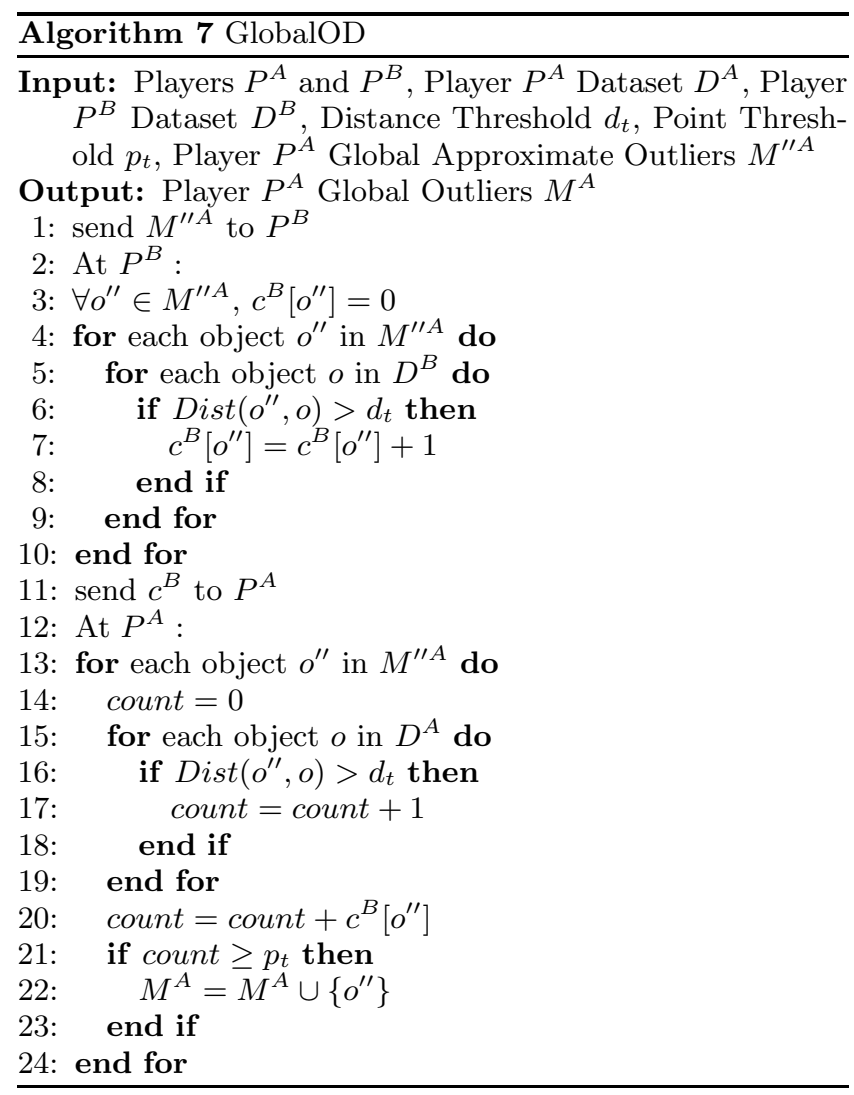

\section{EXPERIMENTS}

All experiments are performed on $\operatorname{Intel}(\mathrm{R})$ Core $i 7 \mathrm{CPU}$ $3.33 \mathrm{GHz}$ machine, using various datasets ${ }^{1}$ listed in Table 1.

Centralized: Table 1 outline the performance of proposed CentralizedOD algorithm. First 3 columns lists the various datasets with respective number of objects and dimensions. The execution time (in seconds) averaged over multiple runs of our algorithm in centralized setting is tabulated in column 4 . We computed the optimal bin threshold $b_{t}$ (listed in column 5) as described in Section 2 for mentioned datasets and ran our algorithm using the same bin threshold. By setting bin threshold to the optimal value, we achieved very low false positive rate as shown in the column 6 . The final phase of removing false positives can be avoided if this false positive rate is acceptable. Even if the false positives needs to be removed, the low false positive rate ensures that the brute force needs to process only few objects. The false negative rate is not mentioned in the table as it is 0 in case of optimal Bin Threshold. In other words, we achieved $100 \%$ detection rate on optimal bin threshold.

For each dataset considered, we computed the percentage of objects for which the FindNeighbors protocol is invoked.

\footnotetext{
${ }^{1}$ All datasets are taken from UCI Machine Learning Repository. http://archive.ics.uci.edu/ml
}

Table 1: Performance of CentralizedOD

\begin{tabular}{|l|r|r|r|r|r|}
\hline Dataset & Objects & Dim & Time & $b_{t}$ & FP \\
\hline Letter & 20000 & 16 & 7.53 & 2 & 0.12 \\
Corel & 68040 & 32 & 32.27 & 20 & 0.09 \\
MiniBooNE & 130064 & 50 & 29.86 & 4 & 0.01 \\
Server & 494021 & 5 & 41.93 & 22 & 0.02 \\
YearPrediction & 515345 & 90 & 193.11 & 4 & 0.03 \\
\hline
\end{tabular}

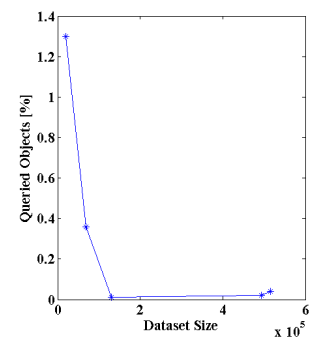

(a)

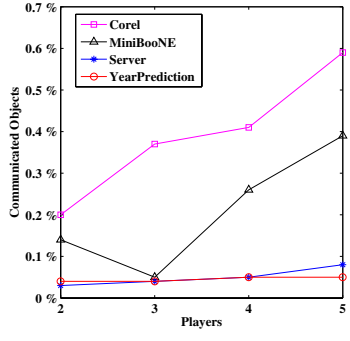

(b)
Figure 1: (a) Queried Objects $N_{q}$ (b) Communication Cost in Horizontal Distribution

The results are shown in Figure 1(a). For small datasets the percentage of queried objects is close to $1 \%$, but as the dataset gets larger this value keeps decreasing rapidly which is evident from the figure.

Distributed: For the distributed case, we have uniformly distributed all the objects in a dataset among the players. For all datasets, the union of the outliers found at each player is equivalent to the set of outliers returned by the centralized algorithm run on the same dataset. This shows that functionality is preserved in the distributed setting also. We have repeated the experiment by varying the number of players from 1 to 5 and studied the effect on the communication cost. The results are given in Figure 1(b). It can be seen that the percentage of the communicated objects is indeed very less.

\section{CONCLUSIONS}

In this paper, we have proposed an approximate algorithm for distance based outlier detection. Our approach uses the Locality sensitive hashing (LSH) to achieve an efficient pruning technique. Further, we have extended our scheme to horizontally distributed setting and proved it to be highly efficient in terms of the communication cost.

\section{REFERENCES}

[1] P. Indyk and R. Motwani. Approximate nearest neighbors: towards removing the curse of dimensionality. In STOC, pages 604-613, 1998.

[2] E. M. Knorr and R. T. Ng. Algorithms for mining distance-based outliers in large datasets. In $V L D B$, pages 392-403, 1998.

[3] S. Ramaswamy, R. Rastogi, and K. Shim. Efficient algorithms for mining outliers from large data sets. SIGMOD Rec., 29:427-438, 2000.

[4] Y. Wang, S. Parthasarathy, and S. Tatikonda. Locality sensitive outlier detection: A ranking driven approach. In Proc. ICDE 2011, 2011. 